\title{
Особенности МДП-структур с фторидом самария на кремниевых и германиевых подложках
}

\author{
(C) М.Б. Шалимова, Н.В. Сачук \\ Самарский национально-исследовательский университет, \\ 443011 Самара, Россия \\ ฯ E-mail: shamb@ssau.ru; sersebroxx@yandex.ru
}

(Получена 26 марта 2018 г. Принята к печати 10 мая 2018 г.)

\begin{abstract}
Электрофизические характеристики кремниевых и германиевых МДП-структур с диэлектрической пленкой $\mathrm{SmF}_{3}$, а также их деградация в результате воздействия электрических полей хотя и подобны, но имеют ряд особенностей. Механизм токопрохождения во всех исследованных структурах описывается степенной зависимостью. Интерфейсные ловушки создают заряд электрически активных ловушек, который изменяется при вольт-емкостных измерениях, и заряд неактивных ловушек, который остается неизменным. На поверхности $n$-Ge этот заряд отрицательный, на поверхности кремния $n$ - и $p$-типа соответствующий заряд положительный. Плотность заряда ловушек в объеме фторида самария лежала в интервале от $-0.2 \cdot 10^{-8}$ до $0.6 \cdot 10^{-8} \mathrm{~K} / \mathrm{cm}^{2}$ и в большинстве случаев была пренебрежимо мала по сравнению с зарядом интерфейсных ловушек.
\end{abstract}

DOI: $10.21883 /$ FTP.2019.02.47106.8873

\section{1. Введение}

Интерес к устройствам на основе структур металл-диэлектрик-полупроводник (МДП) обусловлен многообразием физических явлений, наблюдающихся в таких структурах, и многообразием их функциональных возможностей. Они могут использоваться для конденсаторных, транзисторных приложений, в качестве приборов с зарядовой связью, в качестве мемристоров (устройств, основанных на резистивном переключении).

При продвижении производства кремниевых МДПустройств в субмикронный масштаб возникают трудности, вызванные как физическими ограничениями материала кремния, так и материалов затворного диэлектрика. При пропорциональном уменьшении всех габаритов МДП-структуры ток утечки через затворный диэлектрик возрастает до недопустимых значений. Для увеличения физической толщины слоя диэлектрика при неизменной емкости слой $\mathrm{SiO}_{2}$ заменяется материалом с бо́льшим значением диэлектрической проницаемости (high- $k$ диэлектрики).

Все более привлекательным материалом для каналов полевых транзисторов становится германий, с большей, чем у $\mathrm{Si}$ электронной и дырочной подвижностями. Ключевой проблемой является интеграция $\mathrm{Ge}$ в качестве альтернативного полупроводника для высокоскоростных МДП-устройств и сопутствующего диэлектрика с высокой диэлектрической проницаемостью (high- $k$ ) в качестве материала затвора.

Несмотря на тесную связь между химией границы раздела и электрическим ответом, мало внимания было уделено сравнительному изучению электрического отклика МДП-конденсаторов на кремниевых и германиевых подложках. Известно, что граница раздела $\mathrm{Ge} / \mathrm{GeO}_{2}$, как правило, считается более дефектной, чем граница раздела $\mathrm{Si} / \mathrm{SiO}_{2}$. На электрическое качество $\mathrm{GeO}_{2}$ также может повлиять экспозиция на воздухе и тепловые ступени процесса, потому что $\mathrm{GeO}_{2}$ гигроскопичен и термически неустойчив. Тем не менее считается, что термический рост тонкого промежуточного слоя $\mathrm{GeO}_{2}$ $(k=4.5-5.8)$ является одним из лучших решений для реализации высокой производительности МДП-транзисторов на германии [1].

Внедрение фтора (F) в high- $k$ затворный диэлектрик показало, что он улучшает характеристики устройства и надежность на подложке $\mathrm{Si}$ [2] и подложке $\mathrm{Ge}$ [3]. В работе [2] фтор внедряли в high- $k$ затворный диэлектрик путем отжига в среде $\mathrm{F}_{2}$ в присутствии УФ-излучения, что приводило к пассивации дефектов в объеме high- $k$ диэлектрика и на границе раздела high- $k / \mathrm{Si}$. Авторы работы [4] вводили $\mathrm{F}$ в затворные диэлектрики c помощью имплантации $\mathrm{F}$ с последующим отжигом. Фтор может быть внедрен плазменной обработкой $\mathrm{CF}_{4}$ до или после осаждения затворного диэлектрика $[5,6]$. Было показано, что F имеет тенденцию к сегрегации на границах раздела $\mathrm{HfO}_{2} / \mathrm{SiO}_{2}$ и $\mathrm{SiO}_{2} / \mathrm{Si}$ и пассивирует кислородные вакансии и интерфейсные ловушки путем формирования более сильной связи $\mathrm{Hf}-\mathrm{F}$ и $\mathrm{Si}-\mathrm{F}$. Аналогичный механизм свойствен подложке Ge: образование связей $\mathrm{Ge}-\mathrm{F}$ и $\mathrm{Hf}-\mathrm{F}$ на границе раздела high- $k / \mathrm{Ge}$ и в затворном диэлектрике $\mathrm{HfO}_{2}$ приводит к уменьшению интерфейсных и объемных ловушек [3].

В настоящей работе представлено сравнительное изучение влияния затворного диэлектрика $\mathrm{SmF}_{3}$ на электрофизические характеристики МДП-структур на основе германия и кремния, в том числе с точки зрения деградации их электрофизических свойств при электрической перегрузке (электроформовке).

\section{2. Экспериментальные образцы}

Для экспериментальных образцов в качестве подложек использовались монокристаллические пластины 
германия $n$-типа ориентации (111) с удельным сопротивлением 2 Ом · см и кремния $n$ - и $p$-типа проводимости с удельным сопротивлением 5 Ом · см ориентации (111) и 4.5Ом · см ориентации (100) соответственно. Пленки фторида самария получались методом термического распыления в вакууме порошкообразного $\mathrm{SmF}_{3}$ на подогретые до $\sim 300^{\circ} \mathrm{C}$ подложки германия и кремния. Толщина слоя фторида самария, наносимого на полупроводниковую подложку, варьировалась от $d=0.1$ до 0.4 мкм. Оптимальными для изучения процессов деградации в МДП-структуре методом электроформовки являются толщины 0.2-0.3 мкм. Впоследствии на фторид самария наносились алюминиевые контакты диаметром 0.5-0.7 мм. На всех подложках пленка фторида самария имела поликристаллическую структуру и стехиометрический состав. Диэлектрическая проницаемость кристаллов $\mathrm{SmF}_{3}$, по данным литературных источников, может лежать в диапазоне 10-100 [7]. Измеренная диэлектрическая проницаемость пленок фторида самария, изготовленных в данной работе, составила $k=11-11.5$.

Во всех исследованных структурах, как на кремниевых, так и на германиевых подложках, наблюдается явление бистабильного переключения проводимости, или резистивное переключение. В исходном состоянии МДП-структуры обладают высоким сопротивлением $\sim 10^{11}$ Ом и практически не выпрямляют электрический ток. Образцы находятся в высокоомном состоянии, пока электрическое напряжение, полярность которого соответствует обеднению поверхности полупроводника основными носителями заряда, не превысит некоторое пороговое значение $0.5-4 \mathrm{MB} / \mathrm{cm}$. Это обычно достигается приложением поля электрического пробоя в режиме развертки тока - напряжения при соответствующем ограничивающем токе. При превышении порогового напряжения происходит резкое уменьшение сопротивления образца и переключение его в низкоомное состояние $\left(10^{4}-10^{5} \mathrm{OM}\right)$. Полученное состояние устойчиво и сохраняется при отключении питания. При этом была использована возможность многократного переключения структур из диэлектрического состояния в проводящее и обратно. После каждого цикла переключения структура возвращается в диэлектрическое состояние немного измененной, в частности, с новым током утечки, новой плотностью поверхностных состояний и новым значением эффективного заряда. Это позволяет использовать явление электроформовки в качестве метода исследования деградации параметров и характеристик МДП-структур с фторидом самария.

\section{3. Исследование электрофизических свойств}

Бистабильное переключение в последнее время широко изучается $[8,9]$. Считается, что при первом цикле такого переключения обычно происходит формирование проводящей нити (электроформовка), поэтому для
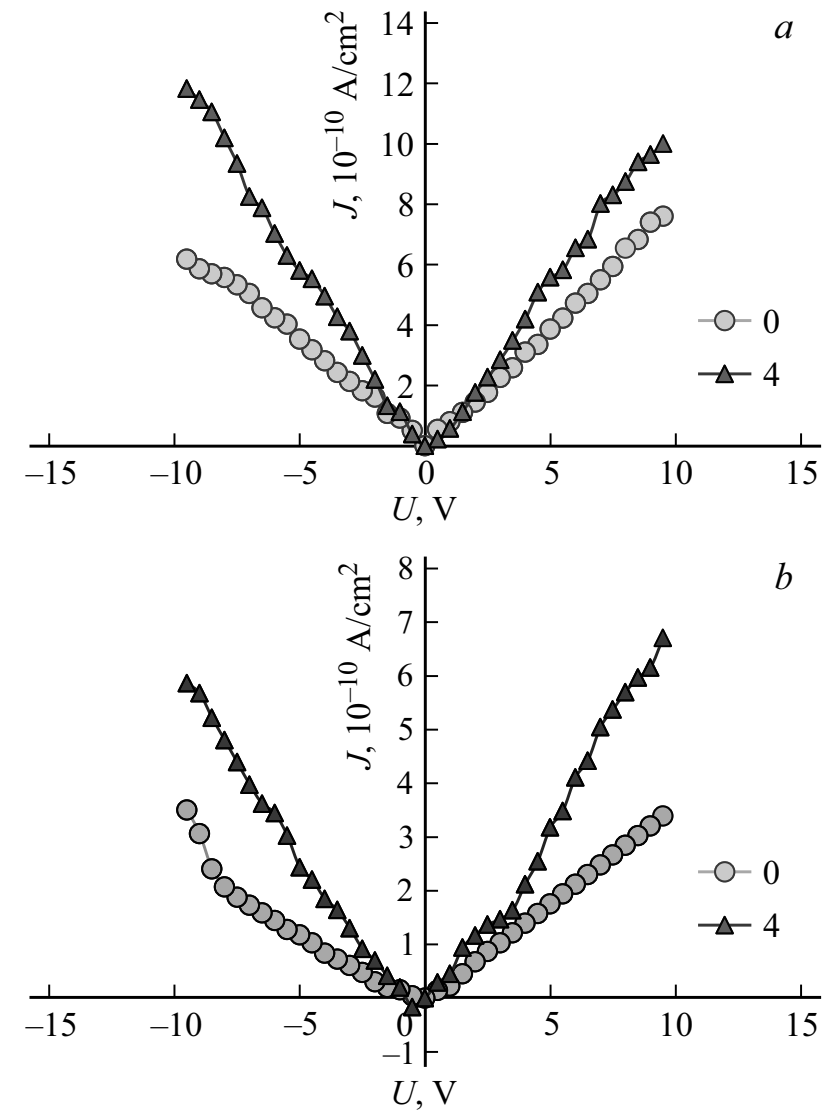

Рис. 1. $a-\mathrm{BAX}$ структуры $\mathrm{Al} / \mathrm{SmF}_{3} / n-\mathrm{Si}$ в исходном состоянии и после нескольких циклов электроформовки, номер цикла указан. $b-\mathrm{BAX}$ структуры $\mathrm{Al} / \mathrm{SmF}_{3} / n$-Ge в исходном состоянии и после нескольких циклов электроформовки, номер цикла указан.

этого процесса требуется бо́льшая напряженность электрического поля. Последние исследования определенно обнаружили, что формирование и обрыв проводящих нитей являются главным переключающим механизмом во многих оксидных и не оксидных системах [8].

Изменение свойств МДП-структуры при последовательных циклах переходов высокоомное/низкоомное состояние можно рассматривать как деградацию электрических свойств затворного диэлектрика и границы раздела диэлектрик-полупроводник при электрической перегрузке. Исследования токов утечки, плотности поверхностных состояний, эффективного заряда помогают изучить механизмы резистивного переключения, механизмы деградации, а также основные механизмы, отвечающие за производительность МДП-устройств.

В настоящей работе представлены вольт-амперные характеристики (BAX) структур $\mathrm{Al} / \mathrm{SmF}_{3} / n-\mathrm{Ge}$, $\mathrm{Al} / \mathrm{SmF}_{3} / n-\mathrm{Si}, \quad \mathrm{Al} / \mathrm{SmF}_{3} / p-\mathrm{Si}$ только в высокоомном состоянии. В состоянии с высоким сопротивлением BAX при обеих полярностях приложенного напряжения практически симметричны, это предполагает, что ток утечки не ограничен электродами. На рис. 1, $a$ показана BAX структуры $\mathrm{Al} / \mathrm{SmF}_{3} / n-\mathrm{Si}$ в исходном высокоомном 
состоянии и после нескольких циклов переключения высокоомное/низкоомное состояние, на рис. $1, b$ аналогичная зависимость для структуры $\mathrm{Al} / \mathrm{SmF}_{3} / n-\mathrm{Ge}$. Характерной особенностью диэлектрической деградации при электрическом стрессе является увеличение тока утечки через диэлектрик.

На рис. 2 представлены прямые ветви ВАХ, построенные в двойных логарифмических координатах для структуры $\mathrm{Al} / \mathrm{SmF}_{3} / n-\mathrm{Si}$ в исходном высокоомном состоянии и после третьего цикла переключения. Эти зависимости достаточно хорошо спрямляются в двойных логарифмических координатах, что указывает на степенной характер зависимости тока от напряжения. Аналогичные степенные зависимости наблюдались в работе [10] для кремниевых МДП-структур с оксидами редкоземельных элементов, и было показано, что соответствующая аналитическая зависимость плотности тока $j$ от напряжения $U$ описывается формулой

$$
j=e G \frac{U^{\gamma}}{d^{2 \gamma-1}} .
$$

Здесь $G$ - некоторая постоянная, зависящая от подвижности носителей заряда в диэлектрике, диэлектрической проницаемости изолятора, эффективной плотности состояний в зоне проводимости диэлектрика, концентрации ловушек вблизи дна зоны проводимости; $e-$ элементарный заряд; $d-$ толщина изолятора; $\gamma-$ показатель степени.

Bce исследованные структуры $\mathrm{Al} / \mathrm{SmF}_{3} / n-\mathrm{Si}$, $\mathrm{Al} / \mathrm{SmF}_{3} / p-\mathrm{Si}$ и $\mathrm{Al} / \mathrm{SmF}_{3} / n-\mathrm{Ge}$ имеют похожие $\mathrm{BAX}$, что указывает на несущественность для механизма токопрохождения того факта, какой конкретно полупроводник контактирует со слоем фторида самария. Показатель степени $\gamma=d \ln (I) / d \ln (U)$ для структур $\mathrm{Al} / \mathrm{SmF}_{3} / n$-Ge принимал значение $\gamma=0.6-2$ при обеих полярностях напряжения в области от нуля до $9 \mathrm{~B}$. Аналогичное значение для структур $\mathrm{Al} / \mathrm{SmF}_{3} / n-\mathrm{Si}$ и $\mathrm{Al} / \mathrm{SmF}_{3} / p-\mathrm{Si}$ составило $\gamma=0.6-2$ и $\gamma=0.7-2$ соответственно.

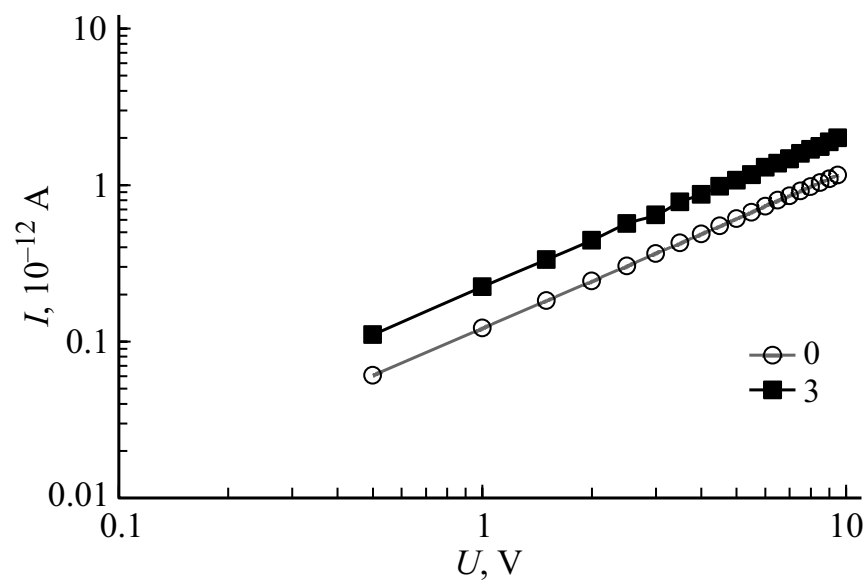

Рис. 2. Прямая ветвь ВАХ структуры $\mathrm{Al} / \mathrm{SmF}_{3} / n$-Si, представленной в исходном состоянии и после нескольких циклов электроформовки, номер цикла указан.

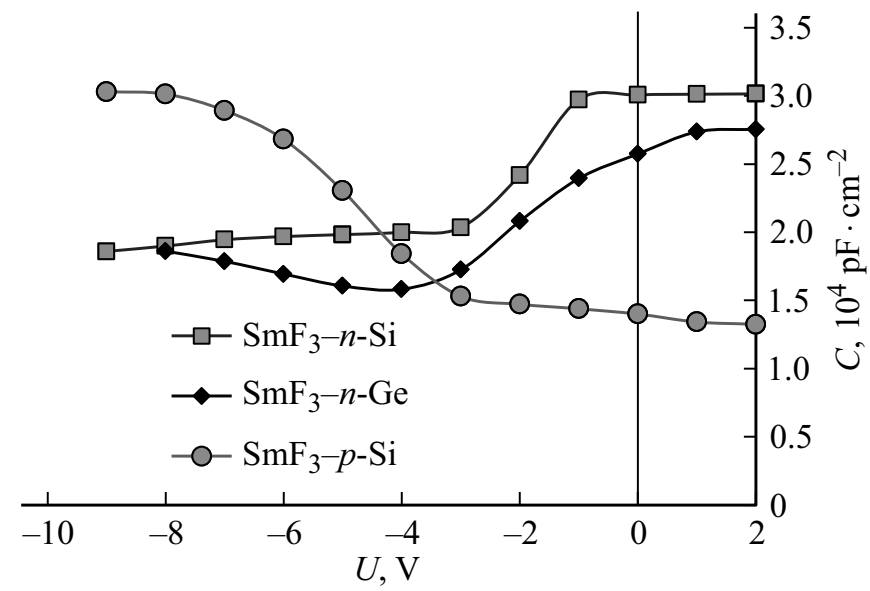

Рис. 3. $C-U$-характеристики исследованных структур в исходном состоянии на частоте 1 МГц.

Наблюдается последовательный рост тока через затворную структуру при увеличении числа циклов переходов высокоомное/низкоомное состояние для всех исследованных структур. Напряженность электрического поля при электроформовке $0.5-4 \mathrm{MB} / \mathrm{cm}$ недостаточна для того, чтобы привести к повреждению горячими носителями объемного диэлектрика $(6 \mathrm{MB} / \mathrm{cm})$. Таким образом, анализ ВАХ указывает на то, что в нашем случае механизм проводимости определяется электрическими свойствами диэлектрика рядом с границей раздела, например, такими, которые связаны с существованием ловушек и их энергетических уровней. Вторая причина связана с тем, что при обратном переключении в высокоомное состояние не происходит полной гомогенизации пленки, на месте канала проводимости остаются локальные высокопроводящие включения, либо остается канал проводимости определенной длины. Фактически это приводит к изменению толщины слоя диэлектрика $d$ в формуле (1), при этом ток растет.

Вольт-емкостные характеристики $(C-U)$ дают информацию об электрически активных поверхностных состояниях и о зарядовом состоянии МДП-структур. На рис. 3 данные характеристики показаны в исходном высокоомном состоянии на частоте 1 МГц для всех исследованных структур. Можно заметить, что из-за более высокой подвижности носителей заряда в германии время их ответа уменьшается, это приводит к росту емкости в инверсии для структуры $\mathrm{Al} / \mathrm{SmF}_{3} / n-\mathrm{Ge}$, что обычно характерно для измерений на более низкой частоте. Это также указывает на то, что диэлектрическое покрытие $\mathrm{SmF}_{3}$ обладает пассивирующими свойствами.

На рис. 4 показаны $C-U$-характеристики структуры $\mathrm{Al} / \mathrm{SmF}_{3} / p$-Si в исходном состоянии (0) и после нескольких циклов электроформовки. После нескольких циклов электроформовки вольт-фарадные характеристики более растянуты по оси напряжений, что указывает на рост плотности поверхностных состояний и изменение напряжения плоских зон. 


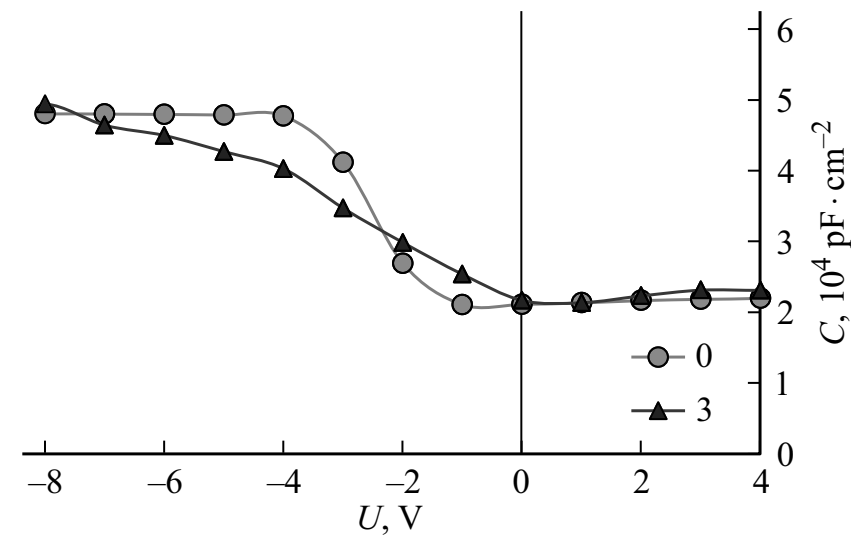

Pис. 4. $C-U$-характеристики структуры $\mathrm{Al} / \mathrm{SmF}_{3} / p$-Si в исходном состоянии и после нескольких циклов электроформовки, номер цикла указан.

Из высокочастотных $C-U$-характеристик, измеренных при комнатной температуре, рассчитывалось напряжение плоских зон $U_{\mathrm{FB}}$, при котором поверхностный потенциал принимает нулевое значение:

$$
U_{\mathrm{FB}}=\Phi_{m s}-\frac{Q_{f}}{C_{D}},
$$

где $\Phi_{m s}$ - разность работ выхода металл-полупроводник, $C_{D}$ - емкость фторида, $Q_{f}-$ эффективный заряд.

$$
Q_{f}=Q_{f}^{*}+Q_{\mathrm{it}},
$$

где $Q_{\mathrm{it}}$ - плотность заряда ловушек на границе раздела фторид самария - полупроводник, $Q_{f}^{*}-$ плотность заряда ловушек в объеме фторида.

Распределение энергетической плотности поверхностных состояний $D_{\text {it }}$ в запрещенной зоне германия рассчитывалось из зависимости поверхностного потенциала от напряжения $U$ по формуле

$$
D_{\text {it }}=\frac{C_{D}}{e^{2}}\left(\frac{d U}{d \phi_{S}}-1\right)+\frac{C_{s}}{e^{2}},
$$

где $C_{s}$ - емкость области пространственного заряда, $\phi_{S}$ - величина поверхностного потенциала. Данный метод дает U-образный вид энергетического распределения $D_{\text {it }}$ в запрещенной зоне германия для исследованных структур. За значение $D_{\text {it }}$ в настоящей работе принималось значение в минимуме распределения.

Зависимость эффективного заряда $Q_{f}$ от плотности поверхностных состояний $D_{\text {it }}$ при многократном переключении высокоомное/низкоомное состояние показана на рис. 5 для МДП-структур с пленками $\mathrm{SmF}_{3}$ на кремниевых и германиевых подложках. Наблюдается тенденция роста положительного заряда при росте плотности поверхностных состояний для структур на основе германия и кремния $n$-типа и рост отрицательного заряда для структур на основе кремния $p$-типа.

Полученный результат согласуется с данными, полученными ранее для оксидов редкоземельных элементов
(Р3Э) на кремниевых подложках [10]. Нужно учитывать, что по оси абсцисс откладывается только энергетическая плотность электрически активных ловушек. Если проводить оценочную экстраполяцию до пересечения данных зависимостей на рис. 5 с осью ординат, то при значении $D_{\text {it }}=0$ получим заряд ловушек, которые не дают ответ при вольт-емкостных измерениях. На поверхности $n$-Ge этот заряд будет отрицательным, что согласуется с теоретическими расчетами, показывающими, что оборванные связи германия на его поверхности выстраивают отрицательный заряд $[11,12]$. Экстраполяция зависимостей $Q_{f}-D_{\text {it }}$ для структур с пленками $\mathrm{SmF}_{3}$ на поверхности кремния $n$ - и $p$-типа дает соответствующий положительный заряд. Возможно, это связано с тем, что на поверхности кремния остается тонкий промежуточный слой $\mathrm{SiO}_{2}$, который содержит положительный заряд, обусловленный наличием кислородных вакансий.

Таким образом, в формуле (3) заряд всех интерфейсных ловушек

$$
Q_{\mathrm{it}}=Q_{\mathrm{it}}^{\mathrm{act}}+Q_{\mathrm{it}}^{\mathrm{pas}},
$$

где $Q_{\mathrm{it}}^{\text {act }}-$ заряд электрически активных ловушек, $Q_{\mathrm{it}}^{\text {pas }}$ заряд неактивных ловушек, который не изменяется при вольт-емкостных измерениях. Анализ показывает, что заряд неактивных ловушек при $D_{\text {it }}=0$ сосредоточен именно на поверхности или вблизи поверхности полупроводника, так как условия формирования диэлектрика во всех случаях были идентичными. Поэтому плотность заряда ловушек $Q_{f}^{*}$ в объеме фторида самария во всех случаях должна быть одинаковой и, согласно оценкам, лежать в интервале от $-0.2 \cdot 10^{-8}$ до $0.6 \cdot 10^{-8} \mathrm{~K} / \mathrm{cm}^{2}$, т. е. в большинстве случаев должна быть пренебрежимо мала по сравнению с зарядом интерфейсных ловушек. Для всех границ раздела $\mathrm{SmF}_{3} /$ полупроводник величина плотности поверхностных состояний изменялась в интервале $(1.5-7.5) \cdot 10^{12}$ эB $^{-1} \cdot \mathrm{cm}^{-2}$, и не наблюдалось

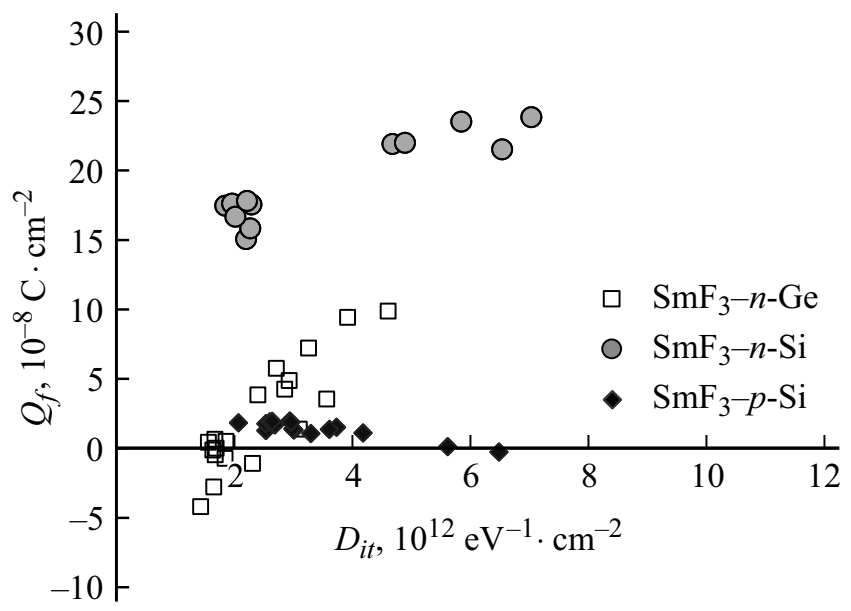

Рис. 5. Изменение эффективного заряда в зависимости от изменения плотности поверхностных состояний для МДПструктур с фторидом самария на основе германия $n$-типа и кремния $n$ - и $p$-типа. 
бо́льшей дефектности германиевой поверхности по сравнению с кремниевой.

\section{4. Заключение}

Результаты эксперимента показывают, что электрофизические характеристики кремниевых и германиевых МДП-структур с диэлектрической пленкой $\mathrm{SmF}_{3}$, а также их деградация в результате воздействия электрических полей хотя и подобны, но имеют ряд особенностей. Механизм токопрохождения во всех исследованных структурах описывается степенной зависимостью. Анализ ВАХ указывает на то, что механизм проводимости определяется электрическими свойствами диэлектрика рядом с границей раздела, например, такими, которые связаны с существованием ловушек и их энергетических уровней. Также на механизм токопрохождения может влиять негомогенность пленки на месте бывшего канала проводимости.

Для всех границ раздела $\mathrm{SmF}_{3} /$ полупроводник величина плотности поверхностных состояний изменялась одинаково при электрической перегрузке, и не наблюдалось бо́льшей дефектности германиевой поверхности по сравнению с кремниевой. Интерфейсные ловушки создают заряд электрически активных ловушек, который изменяется при вольт-емкостных измерениях, и заряд неактивных ловушек, плотность заряда которого остается неизменной. Этот заряд неактивных ловушек сосредоточен именно на поверхности или вблизи поверхности полупроводника, т.е. он определяется непассивированной фторидом поверхностью германия или кремния, так как технология изготовления диэлектрика $\mathrm{SmF}_{3}$ во всех структурах была идентичной. На поверхности $n$-Ge этот заряд отрицательный, на поверхности кремния $n$ - и $p$-типа соответствующий заряд положительный. Плотность заряда ловушек в объеме фторида самария лежала в интервале от $-0.2 \cdot 10^{-8}$ до $0.6 \cdot 10^{-8} \mathrm{~K} / \mathrm{cm}^{2}$ и в большинстве случаев была пренебрежимо мала по сравнению с зарядом интерфейсных ловушек.

Результаты работы могут способствовать оптимизации при выборе материалов границ раздела диэлектрик-полупроводник для устройств на основе кремниевых и германиевых МДП-структур.

\section{Список литературы}

[1] S.N.A. Murad, P.T. Baine, D.W. McNeill, S.J.N. Mitchell, B.M. Armstrong, M. Modreanu, G. Hughes, R.K. Chellappan. Solid-State Electron., 78, 136 (2012).

[2] K. Seo, R. Sreenivasan, P.C. McIntyre, K.C. Saraswat. IEEE Electron Dev. Lett., 27, 821 (2006).

[3] R. Xie, M. Yu, M.Y. Lai, L. Chan, C. Zhu. Appl. Phys. Lett., 92, 163505 (2008)

[4] Y. Mitani, H. Satake, Y. Nakasaki, A. Toriumi. IEEE Trans. Electron Dev., 50, 2211 (2003).

[5] L.K. Chu, R.L. Chu, T.D. Lin, W.C. Lee, C.A. Lin, M.L. Huang, Y.J. Lee, J. Kwo, M. Hong. Solid-State Electron., 54, 965 (2010).
[6] C.X. Li, C.D. Wanga, C.H. Leung, P.T. Lai, J.P. Xu. Microelectron. Eng., 86, 1596 (2009).

[7] S. Rathyamoorthy, S.A.K. Narayandass, B. Calasubramanian, D. Mangalaraj. Phys. Status Solidi A, 117, 495 (1990).

[8] K.M. Kim, D.S. Jeong, C.S. Hwang. Nanotechnology, 22, 254002 (2011).

[9] J.-C. Wanga, Y.-R. Yea, C.-S. Lai, C.-T. Lina, H.-C. Lub, C.-I. Wuc, .-S. Wang. Appl. Surf. Sci., 276, 497 (2013).

[10] М.Б. Шалимова, Н.В. Сачук. ФТП, 49, 1071 (2015).

[11] C.G. Van de Walle, M. Choi, J.R. Weber, J.L. Lyons, A. Janotti. Microelectron. Eng., 109, 211 (2013).

[12] P. Tsipas, A. Dimoulas. Appl. Phys. Lett., 94, 012114 (2009).

Редактор А.Н. Смирнов

\section{Features of MIS structures with samarium fluoride on silicon and germanium substrates}

\section{M.B. Shalimova, N.V. Sachuk}

Samara National Research University, 443011 Samara, Russia

Abstract The electrophysical characteristics of silicon and germanium MIS structures with a $\mathrm{SmF}_{3}$ dielectric film, as well as their degradation as a result of the action of electric fields, although similar, have a number of peculiarities. The mechanism of current transmission in all the structures studied is described by a power law dependence. Interface traps create a charge of electrically active traps, which varies with volt-capacitance measurements, and a charge of inactive traps, which remains unchanged. On the surface of $n$-Ge, this charge is negative, on the $n$ - and $p$-type silicon surfaces the corresponding charge is positive. The charge density of the traps in the volume of samarium fluoride lies in the range from $-0.2 \cdot 10^{-8}$ to $0.6 \cdot 10^{-8} \mathrm{C} / \mathrm{cm}^{2}$, and in most cases was negligibly small in comparison with the charge of interface traps. 